\title{
Elf Thesen zur Zukunft der Unternehmens-Berichterstattung
}

\author{
Extrakt des Beitrags "Grünere und digitalere Bilanzen. Wie moderne \\ Firmen Rechenschaft ablegen: Elf Thesen zur Zukunft der Rech- \\ nungslegung" des Arbeitskreises Externe Unternehmensrechnung der \\ Schmalenbach-Gesellschaft für Betriebswirtschaft e.V.* erschienen \\ in: Frankfurter Allgemeine Zeitung vom 01.11.2021, S. 18.
}

Nach Einschätzung der Mitglieder des Arbeitskreises Externe Unternehmensrechnung (AKEU) der Schmalenbach-Gesellschaft für Betriebswirtschaft e.V. wird die Zukunft der Unternehmensberichterstattung insbesondere durch die Entwicklungen im Nachhaltigkeitsmanagement und der Digitalisierung bestimmt. Der Wandel zu einer Gleichrangigkeit von finanzieller und nicht-finanzieller Berichterstattung, im Sinne einer holistischen Unternehmensberichterstattung, wird nicht nur die Unternehmen, sondern auch deren Abschlussprüfer, das Enforcement, die Investoren, die Mitarbeiter sowie auch die Wissenschaft vor neue Herausforderungen stellen. Diese gilt es unter Zuhilfenahme neuer, digitaler Technologien und einer interdisziplinären Zusammenarbeit zu meistern.

\footnotetext{
AK Externe Unternehmensrechnung | Schmalenbach-Gesellschaft für Betriebswirtschaft e.V.|sg@schmalenbach.org

Mitglieder des Arbeitskreises Dr. Ingrun-Ulla Bartölke, Volkswagen AG | Dr. Rolf Becker, RWE AG | Jens Berger, Deloitte GmbH WPG | Anke Daßler, Evonik Industries AG | Josef Dinger, Fresenius Medical Care AG \& Co. KGaA | Prof. Dr. Michael Dobler, TU Dresden | Dr. Andreas Duhr, thyssenkrupp AG | Martin Edelmann, IASB | Prof. Dr. Brigitte Eierle, Otto-Friedrich-Universität Bamberg | Prof. Dr. Ralf Frank, GISMA Business School I WP Dr. Jens Freiberg, BDO AG WPG | Aurin Gaida, Ruhr-Universität Bochum | Henning Gebhardt I Prof. Dr. Axel Haller, Universität Regensburg I WP Prof. Dr. Sven Hayn, Ernst \& Young GmbH WPG | Prof. Dr. Joachim Hennrichs, Universität zu Köln | Prof. Dr. Christoph Hütten, Universität Mannheim I WP StB Petra Justenhoven, PricewaterhouseCoopers GmbH WPG I Dr. Harald Köster, Henkel AG \& Co. KGaA | Robert Köthner, Daimler AG I WP StB Georg Lanfermann, DRSC | Dr. Guillaume Maisondieu, Deutsche Telekom AG | Ralf Münstermann, METRO AG | Matthias Nawe, E.ON SE | Prof. Dr. Bernhard Pellens, Ruhr-Universität Bochum (AK-Leiter) | Adam Pradela, Deutsche Post AG | Dr. Roman Sauer, Allianz SE | Dr. Wolfgang Sawazki, SALytic Invest AG | Christoph Schauerte, Vonovia SE (AK-Leiter) I Christoph Schlienkamp, GS\&P Kapitalanlagegesellschaft S.A. I Dr. Martin Schloemer, Bayer AG | Prof. Dr. Thorsten Sellhorn, LMU München | Dr. Christopher Sessar, SAP Deutschland SE \& Co. KG | Jonathan Townend, BMW AG | Dr. Jürgen M. Wagner, Siemens AG I Nico Wegmann, Bertelsmann SE \& Co. KGaA | Prof. Dr. Jens Wüstemann, Universität Mannheim I WP StB Christian Zeitler, KPMG AG WPG.
} 
Die Mitglieder des AKEU fassen ihre aktuellen Einschätzungen und Erwartungen in elf Thesen zusammen:

These 1: Der Mindestumfang der Unternehmensberichterstattung wird weiter zunehmen.

These 2: Sowohl die finanzielle Berichterstattung als auch die Nachhaltigkeitsberichterstattung erfolgen nach globalen Standards.

These 3: Die Unternehmensberichterstattung wird holistischer mit Blick auf die gesamtgesellschaftliche Nutzenstiftung.

These 4: Die Unternehmensrechnung und -berichterstattung wird digitaler und modularer werden.

These 5: Unternehmensindividuelle „Key Performance Indicators“ werden in der Unternehmensberichterstattung weiter zunehmen.

These 6: Hoheitliche Regulierung wird die Abschlussprüfung dramatisch verändern.

These 7: Die Nachhaltigkeitsberichterstattung wird künftig dem Enforcement und der Abschlussprüfung genauso wie die finanzielle Berichterstattung unterliegen.

These 8: Das Enforcement wird in der EU hoheitlicher, forensischer und digitalisierter.

These 9: Das Finanzressort wird künftig für die Finanz- und die Nachhaltigkeitsberichterstattung verantwortlich sein.

These 10: Die Mehrzahl der manuellen Tätigkeiten im Finanzbereich erfordert keine klassische Ausbildung und wird in SharedService-Centern erbracht.

These 11: Forschung und Lehre zur Unternehmensberichterstattung fokussieren wieder stärker die drängenden Fragen von Wirtschaft und Gesellschaft. 